\title{
Exploring the Link Between Dry Eye and Migraine: From Eye to Brain
}

This article was published in the following Dove Press journal: Eye and Brain

\author{
Brandon S Baksh ${ }^{1,2}$ \\ Julia Costa Garcia $\mathbb{D}^{1,3}$ \\ Anat Galor (D) ${ }^{1,4}$ \\ 'Bascom Palmer Eye Institute, University \\ of Miami Miller School of Medicine, \\ Miami, FL, USA; ${ }^{2}$ University of Miami \\ Miller School of Medicine, Miami, FL, \\ USA; ${ }^{3}$ Faculdade de Medicina (FMB) da \\ Universidade do Estado de São Paulo \\ (UNESP), Botucatu, Brazil; \\ ${ }^{4}$ Ophthalmology, Miami Veterans Affairs \\ Medical Center, Miami, FL, USA
}

Correspondence: Anat Galor Bascom Palmer Eye Institute, University of Miami Miller School of Medicine, 900 NWI7th Street, Miami, FL, 33I36, USA

Tel + I 305-326-6000

Fax + 305-575-3312

Email agalor@med.miami.edu

\begin{abstract}
Dry eye and migraine are common diseases with large societal and economic burdens that have recently been associated in the literature. This review outlines the link between dry eye and migraine, which may have implications for reducing their respective burdens. We highlight possible shared pathophysiology, including peripheral and central sensitization, as the potential link between dry eye and migraine. Finally, therapies targeting similar pathophysiological mechanisms between dry eye and migraine are discussed.
\end{abstract}

Keywords: dry eye, migraine, brain, sensitization

\section{Introduction}

Awareness of dry eye has increased in recent years including its association with specific diseases, such as migraine headaches. However, our understanding of the link between dry eye and migraine is contingent on what is currently known about them as separate diseases. Specifically, dry eye and migraine are both highly prevalent in the population. The prevalence of dry eye ranges from $5 \%$ to $50 \%$ in the worldwide population, depending on disease definition and population studied, with an overall estimated societal economic burden of $\$ 55.4$ billion in the United States. ${ }^{1}$ As with dry eye, the prevalence of migraine headache is also high. In western countries, the lifetime prevalence of migraine is up to $9.5 \%$ in males and $25 \%$ in females. ${ }^{2}$ The societal economic burden of migraine in the United States is estimated at $\$ 36$ billion. ${ }^{3}$ Thus, migraine headaches and dry eye are important health concerns, and their association warrants further exploration. Understanding shared connections between the two diseases may provide insight into shared pathophysiology and treatments, with a potential decrease in disease morbidity.

To understand the link between dry eye and migraine, we must first define them as separate diseases. Dry eye is defined by the Tear Film and Ocular Surface Society Dry Eye Workshop II as

a multifactorial disease of the ocular surface characterized by a loss of homeostasis of the tear film, and accompanied by ocular symptoms, in which tear film instability and hyperosmolarity, ocular surface inflammation and damage, and neurosensory abnormalities play etiological roles. ${ }^{4}$

The symptoms of dry eye are variable and can include sensations of "dryness", "grittiness", "burning" and "stinging", to name a few. ${ }^{5}$ Individuals may also report that these sensations are spontaneous and/or evoked by wind or light. ${ }^{5}$ Others complain of visual phenomena, such as blurry or fluctuating vision. ${ }^{6}$ Dry eye 
symptoms are commonly assessed in the clinic with specific questionnaires, such as Dry Eye Questionnaire-5 (DEQ-5; range 0-22) ${ }^{7}$ and Ocular Surface Disease Index (OSDI; range, 0-100), ${ }^{8}$ which incorporate many of the above complaints. The DEQ- 5 focuses on intensity and frequency of dryness and discomfort, along with tearing, while the OSDI considers spontaneous and evoked pain, visual complaints, and impact on daily activities. These questionnaires lump responses and generate severity scores, with DEQ-5 scores $\geq 6$ considered indicative of any dry eye symptoms ${ }^{7}$ and scores $\geq 12$ considered severe symptoms. OSDI scores are interpreted as normal $=0-12$, mild $=12-32$, and severe $=33-100 .{ }^{9}$ Of note, these severity scales incorporate a number of different aspects of dry eye to reach a final score, including pain, visual complaints, tearing, and activity limitations. Given that specific symptoms may be driven by different contributors, other questionnaires have been developed to specifically assess for ocular pain complaints, including the Ocular Pain Assessment Survey (OPAS), a 28-question survey, that focuses on intensity of eye pain, non-eye related pain, and aggravating factors, ${ }^{10}$ and the Neuropathic Pain Symptom Inventory-Eye (NPSI-Eye; range, 0-100), which focuses on neuropathic pain features, inquiring about descriptors such as burning pain and evoked pain to wind and light. ${ }^{11}$

In addition to symptoms, clinical signs are also included under the purview of dry eye. The tear film is composed of 2 layers, a thicker muco-aqueous layer that interacts with the corneal epithelium, and a thinner lipid layer that sits on top of the muco-aqueous layer and inhibits its evaporation. ${ }^{12}$ Broadly speaking, dry eye is sub-grouped into categories by dysfunction in these two layers, that is aqueous tear deficient and evaporative dry eye. ${ }^{13}$ Signs of aqueous deficiency include decreased tear volume, assessed by examining the tear meniscus under the slit lamp examination or with the Phenol Red Thread (PRT) test, and reduced tear production, assessed with Schirmer strips (strips of paper placed in the corner of the eye and left in place for 5 minutes, $\mathrm{mm}$ of wetting recorded). The main sign of evaporative deficiency is a rapid tear break up time (TBUT, measured in seconds until a black spot appears in the tear film), which can occur with a dysfunctional lipid layer. However, any tear abnormality, including aqueous deficiency, can result in a rapid TBUT. Furthermore, the sub-types co-exist and individuals may present with both aqueous and evaporative deficiency. Punctate epithelial erosions, which are small disruptions in the corneal epithelium visualized with vital dyes such as sodium fluorescein, rose bengal, or lissamine green, can be seen in both dry eye sub-types and with other ocular surface abnormalities (eg anatomic abnormalities of the eyelid, conjunctivae, or cornea).

The lipid layer is produced by the Meibomian glands (MG) in the upper and lower eyelids. Eyelid abnormalities such as plugging of the MG orifices, MG atrophy, and production of a thicker than normal lipid product (eg abnormal meibum quality) can accompany signs of tear dysfunction. ${ }^{14}$ Point of care tests have also been developed to assess tear composition and inflammation and can specifically evaluate tear osmolarity (TearLab, San Diego) ${ }^{15}$ and ocular surface inflammation (matrix metalloproteinase-9, Inflammadry, Quidel Corporation, San Diego) ${ }^{16}$ in the clinical setting. Some individuals with clinical tear film abnormalities will have high or unstable tear osmolarity levels and/or detectable inflammation on their ocular surface.

A challenge in evaluating dry eye is that the symptoms and signs of disease are often disparate. ${ }^{17,18}$ The presenting symptoms of dry eye can vary even in the same individual and are frequently discordant from the clinical signs and their severity, which can make the diagnosis and management of dry eye difficult. For example, a systematic review of 33 studies assessing associations between dry eye symptoms and signs found that out of 175 individual symptom-sign analyses, only 42 (24\%) were significantly correlated with one another. This study also found that the majority $(129 / 148 ; 87 \%)$ of individual analyses reporting correlation coefficients were in the low-tomoderate range (-0.4 to 0.4$).{ }^{17}$ In addition, the lack of a single objective test with which to evaluate dry eye signs and the low repeatability of tests (eg Schirmer) contributes to the complexity of the disorder.

One of the reasons it is important to screen for dry eye is that dry eye symptoms have a negative impact on individuals' lives as they decrease the ability to work and carry out activities of daily living. ${ }^{19}$ For example, a study recruited 56 individuals with a dry eye diagnosis (International Classification of Diseases, Ninth Revision, ICD-9, codes) for assessment of ophthalmic and quality of life parameters. This study found that individuals with severe dry eye disease (composite score of symptoms [9-level subjective facial expression scale] and signs [Schirmer and corneal surface staining]) had quality of life scores (measured by the time trade-off method) in the range of severe (class III/IV) angina (mean utility 
score, range 0 to 1 , lower values indicate worse quality of life: 0.72 for severe dry eye disease and 0.71 for class III/ IV angina). ${ }^{20}$ In addition, dry eye symptoms have a negative impact on mental health and several studies have linked depression and anxiety to dry eye. ${ }^{21,22}$ Finally, individuals with dry eye have sleep abnormalities. For example, a meta-analysis of 17 studies found that individuals with dry eye symptoms or disease (diagnosed using varying criteria across studies) or primary Sjogren's syndrome had worse sleep quality scores (using Pittsburgh Sleep Quality Index) compared to controls (weighted mean difference $=1.69,95 \%$ confidence interval (CI): $0.82-2.56){ }^{23}$ Taken together, dry eye is a debilitating disease with profound impacts on social functioning and perception of life quality.

Similar to dry eye, migraine is a prevalent condition in the general population. ${ }^{2}$ The International Headache Society (IHS) defines migraine as a "recurrent headache disorder manifesting in attacks lasting 4-72 hours". ${ }^{24}$ Migraine headaches are characterized by unilateral location and pulsating quality and can include nausea, photophobia, and/or phonophobia. Migraine attacks are classified into those with or without aura. Migraine with aura involves reversible prodromal symptoms, such as visual, sensory, or other central nervous system disturbance lasting a few minutes. ${ }^{24}$ Migraine can also be separated into chronic and episodic. Chronic migraine is characterized as occurring $\geq 15$ days per month for three months, which, on at least eight days per month, has features of migraine, while episodic migraine occurs less than 15 days per month. ${ }^{24}$

As with dry eye, migraine symptoms can be debilitating and decrease quality of life. ${ }^{25}$ An observational study of 102 individuals with migraine found disability and health-related quality of life scores were significantly lower than the general population. ${ }^{26}$ Similarly, a retrospective cross-sectional survey study of 80,600 European patients found lower health-related quality of life and decreased work productivity among those with $\geq 4$ monthly migraine headaches compared to nonmigraine controls. ${ }^{27}$ Interestingly, lower quality of life scores among those with migraine closely associate with dry eye symptoms. In a cross-sectional survey-based study of 62 individuals with migraine, visual function (measured via visual functioning questionnaire-25) and overall quality of life (measured via headache impact test-6) correlated with dry eye symptoms (measured via OSDI score) ${ }^{28}$ Together, these data show that both dry eye, migraine, and perhaps their interaction, have significant negative impacts on patient quality of life. Thus, in this review, we explore the association between dry eye and migraine with the goal of illuminating overlapping pathophysiology and potential therapies. To do so, we reviewed recent studies that investigated the relationship between dry eye and migraine.

\section{Methods}

A PubMed search was conducted using the terms "dry eye" AND "migraine". All published scientific articles were considered including original research, metaanalyses, and systematic reviews. All searches were limited to the English language. Eligible articles were reviewed and summarized.

\section{Clinical Associations Between Dry Eye and Migraine Epidemiology of Dry Eye, Migraine, and Their Co-Existence}

Dry eye and migraine are co-morbid. Using survey data from a Korean population-based cross-sectional study of 14,329 participants, the prevalence of migraine and dry eye diagnosis was found to be similar among participants: $24.2 \%$ reported migraine headaches (positive answer to "Do you have, or have you ever experienced migraine [pulsatile pain unilaterally in your head]?"), $22.6 \%$ reported a dry eye diagnosis (positive answer to "Have you ever been diagnosed with dry eye by an ophthalmologist?"), and 37.1\% reported dry eye symptoms (positive answer to "Do your eyes tend to be dry, with a foreign body sensation including itching and burning or sandy feeling lately?"). ${ }^{29}$ Furthermore, the frequency of dry eye diagnosis was found to be higher in those with migraine. Of those with migraine, $14.4 \%$ reported a dry eye diagnosis compared to $8.2 \%$ without migraine, $\mathrm{p}<0.0001$. Similarly, of those with migraine, $22 \%$ reported dry eye symptoms compared to $15.1 \%$ without migraine, $\mathrm{p}<0.0001 .{ }^{29}$ While limitations of this study included the use of data assessed via questionnaires, other studies have reported similar results. In a hospital-based case-control study of 72,969 individuals from University of North Carolina-affiliated hospitals, individuals with migraine and dry eye were identified using International Classification of Diseases, Ninth and Tenth Revision (ICD-9 and ICD-10) codes. The prevalence of a migraine or dry eye diagnosis was $7.3 \%$ and $13.2 \%$, respectively. Again, individuals with migraine had a higher frequency of a co-morbid dry eye 
diagnosis. Of those with migraine, $19.6 \%$ had a dry eye diagnosis compared to $12.7 \%$ without migraine. ${ }^{30}$

Looking at the data as odds ratios (OR), in the population-based questionnaire study of 14,329 Korean individuals, after adjusting for confounders, the presence of migraine was found to increase the odds of a dry eye diagnosis 1.58 fold (95\% confidence interval (CI) 1.341.86) and the odds of dry eye symptoms 1.3 fold (95\% CI, $1.15-1.50){ }^{29}$ In the study of 72,969 individuals from North Carolina, the presence of migraine increased the odds of a dry eye diagnosis 1.42 fold (95\% CI, 1.20-1.68). The association was strongest among women $\geq 65$ years old (OR, 2.47; 95\% CI, 1.75-3.47). ${ }^{30}$

Other studies have investigated the reverse relationship, that is the odds of migraine in individuals with dry eye. In a large Taiwanese study using ICD-9 codes $(n=48,028)$, the presence of a dry eye diagnosis increased the odds of a migraine diagnosis 1.76 fold (95\% CI, 1.57-1.98), after adjusting for co-morbidities. ${ }^{31}$ While these studies suggest a reciprocal relationship between dry eye and migraine, they are limited by their retrospective or cross-sectional nature and reliance on ICD coding and questionnaires for migraine and dry eye diagnosis. This is especially relevant as dry eye is a heterogeneous disease and it is unclear which combination of symptoms and/or signs led to the coded diagnosis. Overall, these studies suggest that dry eye and migraine are common conditions and that individuals with migraine are more likely to have dry eye symptoms and carry a dry eye diagnosis compared to those without. However, a limitation of the studies is that they did not look at dry eye signs and as such, it is difficult to understand what component of dry eye is most closely related to migraine.

\section{Dry Eye Characteristics Among Individuals with Migraine}

To further explore relationships between dry eye and migraine, several smaller studies investigated associations between migraine and dry eye symptoms and signs. In a cross-sectional study of South Florida veterans seen in a dry eye clinic, 31 individuals with migraine (defined via the American Migraine Study/American Migraine Prevalence and Prevention (AMS/AMPP) migraine diagnostic module $)^{32}$ were compared to 219 individuals without migraine. Migraineurs had significantly higher dry eye symptom scores (via OSDI) but similar tear metrics (TBUT, corneal staining, tear production) compared to controls. $^{33}$ Interestingly, NSPI-Eye scores, assessing for neuropathic features of eye pain, were also higher among individuals with migraine compared to controls. These data suggest dry eye symptoms, but not dry eye signs, are related to migraine. This conclusion is supported by other studies, as well. One observational study of Turkish individuals seen in a dry eye clinic compared 33 individuals with migraine to 33 controls. Migraine was diagnosed by different neurologists. Dry eye symptoms were assessed using OSDI and dry eye signs using TBUT, corneal staining, and Schirmer test. Migraineurs had significantly higher dry eye symptoms, lower TBUT, and Schirmer scores, and higher corneal staining compared to controls. ${ }^{34}$ However, Schirmer scores were within normal limits in both groups (mean $>10 \mathrm{~mm} / 5 \mathrm{~min}$ ) and thus the clinical relevance of the differences in values is unclear. Similar findings were reported in another study of 46 Turkish patients with migraine and 50 controls that were assessed for Sjogren's Syndrome, dry eye symptoms (via OSDI), and dry eye signs (TBUT, Schirmer) in a rheumatology clinic. $^{35}$ Migraine was diagnosed by the referring neurologist. In this study, individuals with migraine had significantly higher dry eye symptoms and lower TBUT and Schirmer scores compared to controls, however again, Schirmer results were still within normal limits (mean $>10 \mathrm{~mm} / 5$ min). Another case-control study performed in a United States ophthalmology clinic assessed dry eye symptoms and signs and corneal nerve parameters in 19 individuals with chronic migraine. This study used 30 controls from a normative dataset for corneal nerve comparisons, but no control data were included for dry eye parameters. Chronic migraine was defined by the International Headache Society guidelines. Dry eye symptoms via measured DEQ-5 were abnormal in all subjects (DEQ-5 $>6$ ), but tear parameters were within normal limits among all individuals with chronic migraine (data not reported). ${ }^{36}$ Interestingly, corneal nerve fiber density was significantly lower in individuals with migraine compared to controls $(48 \pm 23$ vs $71 \pm$ 15 fibers $/ \mathrm{mm}^{2}$ ). However, given the lack of standard nomograms for corneal nerve fiber density, the interpretation of this finding is uncertain. Together, these studies point to dry eye symptoms being more closely related to migraine than dry eye signs.

\section{Migraine Characteristics Among Individuals with Dry Eye}

As above, while some studies evaluated dry eye characteristics in individuals with migraine, other studies evaluated 
whether specific migraine characteristics were more closely associated with dry eye. A Turkish study that evaluated 58 individuals with migraine reported that the odds of having dry eye (defined if 2 of 3 criteria met: OSDI $>33$, TBUT $<10$ seconds or Schirmer $<10 \mathrm{~mm} / 5 \mathrm{~min}$ ) were 5.03 times higher in those with migraine and aura compared to those without aura $(95 \% \mathrm{CI}, 1.42-17.83){ }^{37}$ These data suggest that migraine with aura is more closely associated with aspects of dry eye than migraine without aura.

In addition to aura, the lifetime duration of migraine has also been explored in its relationship to dry eye. In the above Turkish study, individuals with a dry eye diagnosis had a longer median lifetime duration of migraine compared to those without a diagnosis (10 vs 6 years, $\mathrm{p}=0.01){ }^{37}$ Similarly, another Turkish study of 46 individuals with migraine (diagnosed by a neurologist) found that migraine lifetime duration correlated with both dry eye symptom severity (OSDI score) $(\mathrm{r}=0.3, \mathrm{p}=0.01)$, tear stability (TBUT: $\mathrm{r}=-0.23, \mathrm{p}=0.05$ ), and tear production (Schirmer: $\mathrm{r}=-0.28, \mathrm{p}=0.01$ ). Of note, the negative correlations imply that longer duration of migraine associated with faster break-up time and lower tear production. ${ }^{35}$ Taken together, these studies suggest that migraine with aura and longer disease duration are associated with aspects of dry eye. However, it is important to note that definitions of dry eye were not uniform among studies, and migraine criteria were not always clearly outlined.

\section{Photophobia is a Feature of Both Dry Eye and Migraine}

Thus far, we have discussed associations between dry eye and migraine. However, the diseases also share a common feature, that is, the presence of photophobia. Although photophobia is variably defined in the literature, in this review, photophobia refers to light-induced neurological symptoms, which usually emerge in the form of (i) increased sensitivity to light or glare, (ii) intensification of headache and (iii) ocular pain or discomfort. ${ }^{38}$ With regards to dry eye, our group reported that $75 \%$ of 236 veterans with dry eye symptoms (DEQ-5 score $\geq 6$ ) reported pain sensitivity to light (defined as score $\geq 1$ on a $0-10$ numerical rating scale (NRS)). ${ }^{39}$ In another study, we found that of 102 South Florida veterans, individuals with persistent dry eye symptoms (DEQ5 score $\geq 6$ over a 2 -year period) were more likely to report photophobia compared to those without persistent symptoms (OR, 15.6; 95\% CI, 2.0 to $123, \mathrm{p}=0.009){ }^{40}$
Our data suggest that photophobia is a common feature in individuals with dry eye symptoms, and in fact, presence and severity of photophobia is the first question on the OSDI.

Photophobia is also a common feature in migraine. In a cross-sectional survey of 6045 respondents in the Migraine in America: Symptoms and Treatment Study, 49.1\% reported photophobia as the "most bothersome symptom. ${ }^{41}$ In a retrospective cross-sectional study of 117 individuals with chronic migraine ( $\geq 15$ headache days/month), $80 \%$ rated their photophobia (via 0-10 NRS) as severe (a score of $\geq 7 / 10$; mean $7.91 \pm 2.05)$. $^{42}$ Together, the data demonstrate that photophobia is a feature of both dry eye and migraine. The presence of photophobia in both diseases has implications for shared pathophysiology and treatments as discussed later in the review.

\section{Neural Pathways Mediating Photophobia}

Studies have explored the neural circuitry underlying photophobia, both in the context of dry eye ${ }^{43}$ and migraine. ${ }^{44}$ One pathway involves light-evoked signals in rod and cone cells that are transmitted to retinal ganglion cells (RGC) via amacrine and bipolar cells. Some signals in RGCs are transmitted to the olivary pretectal nucleus (OPN), then to the superior salivatory nucleus, and subsequently to the sphenopalatine ganglion, which stimulates parasympathetic-mediated vasodilation of ocular $^{45}$ and dural ${ }^{38}$ vessels that are innervated by trigeminal afferents. Trigeminal signals subsequently travel to the trigeminal nucleus caudalis, posterior thalamus, and cortical structures (Figure 1). ${ }^{38}$ Evidence for this pathway comes from immunocytochemistry experiments in rats that demonstrated light-evoked neuronal activity in the trigeminal brainstem, which was reduced after intravitreal injection of norepinephrine. These data suggest that constriction of ocular blood vessels by norepinephrine plays a role in light-evoked neuronal activity, thus implicating ocular vasculature in the trigeminal brainstem pathway of photophobia. ${ }^{45}$ A mouse study similarly found a trend for reduced blue-light aversion behavior (measured by amount of time mice spent in the illuminated portion of a box) after intravitreal injection of norepinephrine, but the reduction did not reach statistical significance. $^{46}$

A second neural pathway involves light-sensitive neurons in the posterior thalamus, specifically the lateral 


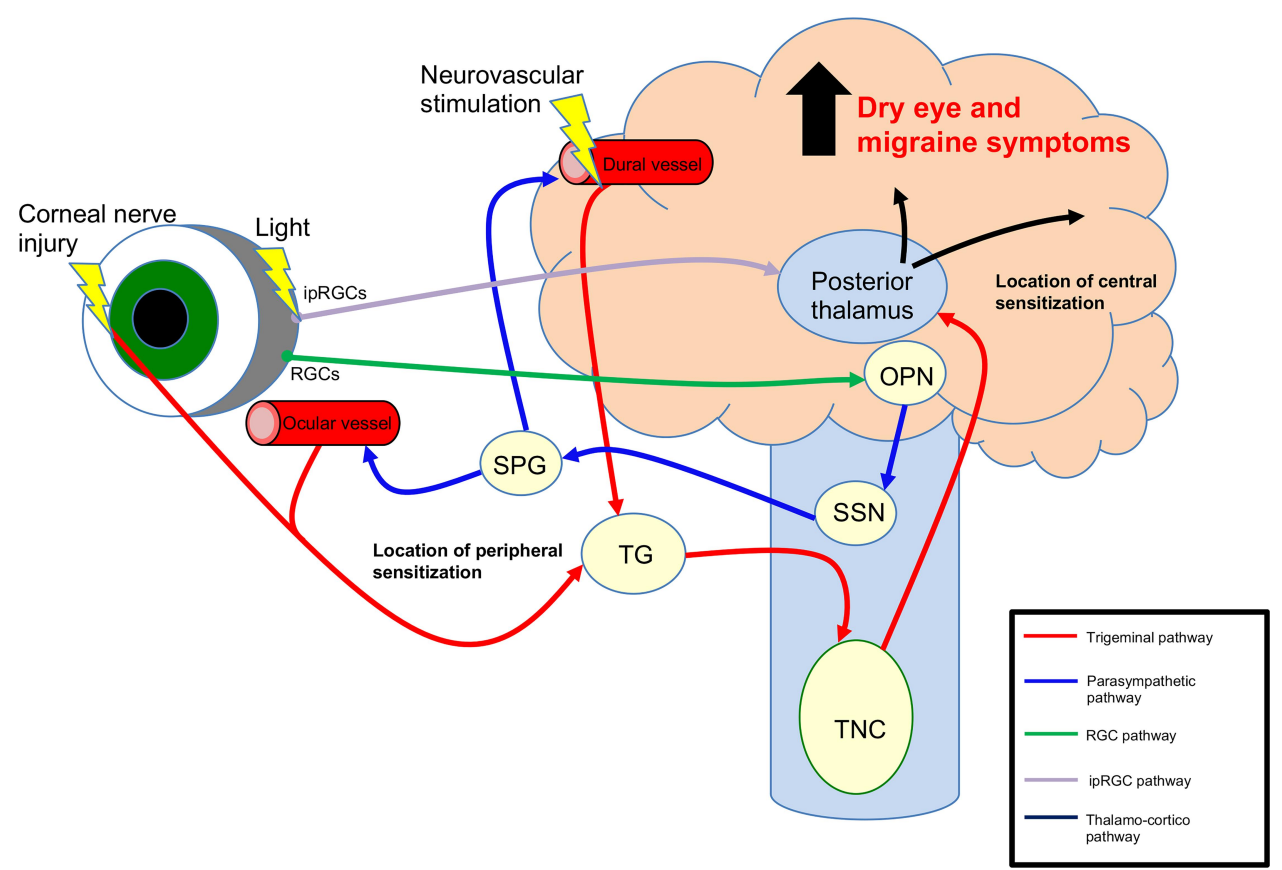

Figure I Selected photophobia neural pathways in dry eye and migraine. Light evokes signals from rod and cone cells that are transmitted via amacrine and bipolar cells (not shown) to retinal ganglion cells (RGC), which project to the olivary pretectal nucleus (OPN, green line). Blue line: parasympathetic signals travel from the OPN to the superior salivatory nucleus (SSN), then to the sphenopalatine ganglion (SPG), and ocular and dural vessels to mediate vasodilation. Red line: afferent trigeminal signals from cornea (stimulated by corneal disruptions), ocular vessels, and dural vessels (stimulated by vasodilation) travel to the trigeminal ganglion (TG) then to the trigeminal nucleus caudalis (TNC) and finally the posterior thalamus. Alternatively, light-evoked signals from intrinsically photosensitive RGCs (ipRGC) travel directly to the posterior thalamus (purple line). Black line: signals from the posterior thalamus travel to somatosensory and visual cortices to mediate dry eye and migraine symptoms. Note other pathways of photophobia that involve the hypothalamus and retinal rod and cone cells are not depicted.

posterior (LP) and posterior nuclei (PO), ${ }^{38}$ which receives input from both intrinsically photosensitive RGCs (ipRGC) and dural trigeminal afferents, and subsequently send signals to somatosensory and visual cortices (Figure 1). ${ }^{38,47}$ Evidence for this pathway comes from a rat study using electrophysiologic and histopathologic techniques which demonstrated that cell bodies and dendrites of dura- and light-sensitive neurons in the posterior thalamus were in close apposition to axons originating from ipRGCs. ${ }^{48}$ Other studies have further connected the posterior thalamic nuclei to photophobia. A mouse model found that stimulation of posterior thalamic nuclei (LP and PO nuclei) by optogenetics or injection of calcitonin gene-related peptide (CGRP) $)^{49}$ triggered light aversive behavior. ${ }^{50}$ Beyond these two pathways, other postulated, but less well studied pathways in photophobia involve the hypothalamus, retinal rod and cone cells, and the iris. ${ }^{47,51,52}$

\section{Dry Eye and Migraine Share Underlying Pathophysiology}

The clinical overlap between dry eye symptoms and migraine, including the presence of photophobia, suggests pathophysiological links between them. One unifying theory is that dry eye symptoms and migraine involve abnormal peripheral trigeminal nerve activation with subsequent peripheral and central sensitization. Peripheral sensitization is defined as "increased responsiveness and reduced threshold of nociceptive neurons in the periphery to the stimulation of their receptive fields" ${ }^{\prime \prime 53}$ and below we focus on corneal peripheral nerve abnormalities that have been described in dry eye and migraine. Central sensitization is defined as "increased responsiveness of nociceptive neurons in the central nervous system to their normal or subthreshold afferent input" ${ }^{\prime 53}$ and below we focus on changes in secondary and tertiary nerves that connect corneal afferents to higher cortical areas.

\section{Tests Used to Evaluate Nerve Abnormalities in Dry Eye and Migraine in Animal Models and Humans}

In animals, peripheral nerve function is often evaluated via electrophysiological recordings of polymodal $(\sim 70 \%)$, mechano- (10-15\%), and cold thermoreceptors (10-15\%) within corneal nerves or via recordings in ciliary nerves. ${ }^{54-56}$ Polymodal nociceptors respond to mechanical force, heat, chemical irritants and inflammatory mediators, ${ }^{57}$ mechanoreceptors to mechanical forces, and cold thermoreceptors to 
temperature drop and changes in tear osmolarity. ${ }^{58}$ Electrophysical recordings are also used to evaluate central nerve function along trigeminal pathways, such as in the trigeminal nucleus caudalis. ${ }^{59}$ Corneal sensitivity is also tested in animals with von Frey filaments, where increasing force is used to contact the central cornea until a blink-response is elicited. ${ }^{55}$

In humans, electrophysiological recordings of corneal and central nerves are not feasible. As such, clinicians have developed several tests that evaluate corneal nerve pathway function. In the clinic, corneal sensitivity is typically qualitatively checked with a cotton tip or dental floss (rated as $0=$ absent, $1=$ reduced, $2=$ normal, 3 , increased). In the research arena, corneal sensitivity can be quantitatively assessed using a Cochet-Bonnet esthesiometer where a nylon monofilament $6 \mathrm{~cm}$ in length is used to contact the ocular surface and then retracted in $0.5-\mathrm{cm}$ increments until corneal sensation is felt. For this test, a higher result corresponds to a higher corneal sensitivity. ${ }^{60}$ Alternatively, a Belmonte esthesiometer utilizes a non-contact air jet to provide the stimulus, which can either be mechanical (variable airflow), thermal (hot or cold pulses), or chemical (varying $\mathrm{CO} 2$ concentrations). In contrast to CochetBonnet, lower values with the Belmonte esthesiometer correspond to increasing sensitivity. The presence of hypo- or hyper-sensitivity suggests abnormalities in the corneal nerve pathway, although it is not possible to determine if the abnormality is in peripheral and/or central nerves. Overall, a wide range of corneal sensitivity values has been found in individuals with and without dry eye. ${ }^{61}$ One study of 403 individuals with dry eye symptoms (DEQ5 $\geq 6$ ) found a mean corneal mechanical detection threshold (using Belmonte esthesiometer) of $87 \pm 46 \mathrm{~mL} /$ min, with a 10th percentile of $40 \mathrm{~mL} / \mathrm{min}$ and a 90th percentile of $145 \mathrm{~mL} / \mathrm{min}$. Twenty-four percent of individuals had values that fell at or outside this range, 13\% $(\mathrm{n}=51)$ were hypersensitivity $(\leq 40 \mathrm{~mL} / \mathrm{min})$ and $11 \%$ ( $\mathrm{n}=46)$ hyposensitive $(\geq 145 \mathrm{~mL} / \mathrm{min}){ }^{61}$

Peripheral nerve structure can be assessed using in vivo confocal microscopy (IVCM). IVCM images can be used to examine corneal subbasal nerves for density, branching, beading, tortuosity, and abrupt termination with swelling (termed microneuroma). ${ }^{62}$ IVCM, however, has limitations in that it lacks built-in software to analyze nerve parameters, there are no normative databases with which to compare values across populations, it provides a small field of view, and it is difficult to scan the exact same location over time. ${ }^{63}$
In humans, certain symptom profiles suggest central abnormalities including the presence of allodynia (pain due to a stimulus that does not normally provoke pain, ${ }^{53}$ such as with light), hyperalgesia (increased pain from a stimulus that normally provokes pain, ${ }^{53}$ such as with wind) and expansion of the receptive fields (such as pain to light touch of the periocular skin). ${ }^{43,64}$ The proparacaine challenge is another clinical test used to detect central abnormalities. Individuals are first asked to rate their ocular pain intensity (typically on a $0-10$ scale) immediately prior to placement of topical anesthetic. After one drop is instilled in each eye and 30 seconds to 2 minutes have passed (different investigators use different time periods), ocular pain is reassessed. Elimination of pain suggests nociceptive or peripheral contributors to pain while persistence of pain suggests central or non-ocular contributors. A limitation of this test is that it is not informative if no pain is present at the start of testing. In the research arena, brain functional studies ${ }^{65}$ and quantitative sensory testing have been used to identify central abnormalities in trigeminal pathways. ${ }^{66}$

\section{Abnormalities in Peripheral Nerves Have Been Detected in Dry Eye and Migraine}

The literature suggests that both dry eye symptoms and migraine pain are driven in part by peripheral sensitization. ${ }^{67,68}$ In dry eye, peripheral injury and activation may result from a number of sources including chronic epithelial disruptions, high tear osmolarity, ocular surface inflammation, and/or surgically induced nerve injury (eg refractive surgery). ${ }^{67}$ On the other hand, initiators of peripheral nerve injury in migraine remain controversial. $^{69}$

Electrophysiology studies have detected corneal nerve abnormalities in dry eye. In a guinea-pig model of aqueous tear deficiency using lacrimal gland excision, changes in peripheral nerve function were detected in mechanoreceptor spontaneous activity at 1 week postsurgery $(0.30 \pm 0.22$ vs $0.02 \pm 0.02 \mathrm{impulses} /$ second, $\mathrm{p}<0.05)$ and cold-thermoreceptor spontaneous activity at 4 weeks post-surgery $(13.22 \pm 1.00$ vs $10.27 \pm 0.78$ impulses/second, $\mathrm{p}<0.05)$ compared to sham controls. Furthermore, a change in cold-thermoreceptor thresholds was observed 4 weeks post-surgery (32.42 \pm 0.14 vs $29.87 \pm 0.35{ }^{\circ} \mathrm{C}$, respectively, $\left.\mathrm{p}<0.05\right)$ compared to controls, indicating increased sensitivity to cooling. ${ }^{56}$ In a mouse model, lacrimal gland excision resulted in an 
increase in spontaneous ciliary nerve activity compared to sham controls $(86.8 \pm 7.6$ vs $43.4 \pm 4.9 \mathrm{impulses} / \mathrm{sec}$, $\mathrm{p}<0.001)$. Concomitantly, corneal mechanical thresholds decreased (implying increased sensitivity) compared to sham controls $(0.012 \pm 0.001$ vs $0.028 \pm 0.002 \mathrm{~g}$, $\mathrm{p}<0.0001) .^{70}$ Together, the studies demonstrate that the initiation of aqueous tear deficiency causes a change in corneal nerve function, manifesting as hypersensitivity. Unfortunately, corneal nerve electrophysiology studies in migraine animal models are lacking.

Alterations in corneal nerves structure and function have also been reported in various dry eye populations compared to controls. Overall, most studies have reported decreased corneal nerve density and sensitivity in individuals with aqueous tear deficiency and Sjögren's syndrome but not in individuals with evaporative dry eye. ${ }^{62}$ For example, an Italian study examined corneal nerves in 39 individuals with symptomatic aqueous tear deficiency (low TBUT, corneal staining, low Schirmer) compared to 30 controls. They found significantly lower corneal nerve fiber density and length, but higher width in the dry eye vs control group (respectively, $20.5 \pm 8.7$ vs $25 \pm 6.7 \mathrm{n} / \mathrm{mm}^{2}$, $\mathrm{p}=0.008 ; 12.6 \pm 4.4$ vs $14.5 \pm 2.9 \mathrm{~mm} / \mathrm{mm}^{2}, \mathrm{p}=0.02 ; 0.021$ \pm 0.001 vs $\left.0.019 \pm 0.001 \mathrm{~mm} / \mathrm{mm}^{2}, \mathrm{p}<0.001\right){ }^{71}$ For corneal sensitivity, an American study of 33 individuals with symptomatic aqueous tear deficiency (OSDI $>20$, TBUT $\leq 7$ seconds, tear meniscus height $<220 \mu \mathrm{M}$ ) found decreased sensitivity (measured via Cochet-Bonnet) compared to 10 healthy controls $(3.6 \pm 1.6$ vs $5.5 \pm 0.83 \mathrm{~cm}$, $\mathrm{p}<0.05)$. Similar to density, individuals with other dry eye sub-types (Meibomian gland dysfunction and conjunctivochalasis) did not have differences in corneal sensitivity compared to controls. ${ }^{72}$ Together, the above studies suggest that individuals with aqueous tear deficiency have lower nerve densities and sensitivity than controls, but that these differences are not as robust in other dry eye sub-types.

Corneal nerve alternations have also been documented in migraine. A Chinese study examined corneal nerves in 10 individuals with episodic migraine and 10 controls. Corneal nerve branching and tortuosity were significantly increased in individuals with migraine compared to controls $\left(91 \pm 13.8\right.$ vs $75 \pm 14.2$ branches $/ \mathrm{mm}^{2}, \mathrm{p}=0.03$ and $2.3 \pm 4.6$ vs $1.6 \pm 0.5, \mathrm{p}=0.01$, respectively). ${ }^{73}$ Photophobia has also been linked to peripheral corneal nerve abnormalities. In a prospective Indian study, individuals with chronic migraine and photophobia $(n=36)$ had significantly lower subbasal nerve parameters, including corneal nerve fiber length $(14.8 \pm 4.0$ vs $18.1 \pm 3.3 \mathrm{~mm} /$ $\left.\mathrm{mm}^{2}, \mathrm{p}=0.007\right)$, compared to those with migraine but no photophobia $(n=24) .^{74}$

Individuals with migraine have also been found to have increased corneal sensitivity compared to controls. One Turkish study compared 58 individuals with chronic migraine to 30 controls. Corneal sensitivity (measured by Cochet-Bonnet) in the nasal region was higher (increased sensitivity) in the migraine vs control group [median (IQR); $5.5(5.25-6.0)$ vs $5.37(5.0-5.75) \mathrm{cm}, \mathrm{p}=0.02]$. Interestingly, in individuals with unilateral migraine, corneal sensitivity was higher in the affected vs unaffected side (median (IQR); 5.4 (5.0-5.7) vs 5.3 (5.0-56.5), $\mathrm{p}=0.049) .^{75}$ The data on sensitivity, however, are limited in that the Cochet-Bonnet can only measure sensitivity up to $6 \mathrm{~cm}$ and most healthy individuals can detect the filament when fully extended. No studies have evaluated corneal sensitivity in migraine with Belmonte esthesiometry which has a wider testing range. Overall, while not as robust as for dry eye, studies demonstrate that individuals with migraine have changes in their corneal nerve structure and function compared to controls.

\section{Abnormalities in Central Nerves Have Been Detected in Dry Eye and Migraine}

The literature suggests that both dry eye symptoms and migraine pain are driven in part by central sensitization. Given that corneal nerve fibers project to the trigeminal brainstem region, studies have used this region to investigate central nerve changes in dry eye. ${ }^{70}$ In a lacrimal gland excision mouse model, an increase in spontaneous firing rate of trigeminal subnucleus interpolaris/caudalis $(\mathrm{Vi} / \mathrm{Vc})$ neurons was noted compared to sham controls $(6.4 \pm 1.9$ vs $2.9 \pm 1.4 \mathrm{~Hz}, \mathrm{p}<0.05)$. Additionally, periocular cutaneous receptive field areas of $\mathrm{Vi} / \mathrm{Vc}$ and $\mathrm{Vc} / \mathrm{C} 1$ units were significantly enlarged compared to sham controls. ${ }^{59}$ These data suggest that aqueous tear deficiency can lead to central nerve abnormalities.

As with dry eye, central nerve abnormalities have been demonstrated in migraine. In a rat model of migraine using dural stimulation with an "inflammatory soup" (i.e histamine, serotonin, bradykinin), electrophysiologic recordings from trigeminovascular neurons in the posterior thalamus showed an increased firing rate and increased magnitude of responses to pressure, pinch, cephalic and extracephalic brush after dural stimulation compared to baseline. In contrast, control animals (dura stimulated 
with fluid) showed no change in responsiveness after stimulation compared to baseline. ${ }^{76}$

Central abnormalities have also been noted in humans with dry eye and migraine. With regards to dry eye, a cross-sectional study of 224 South Florida veterans with dry eye symptoms (DEQ-5 $\geq 6$ ) found that $18(41 \%)$ had persistent ocular pain (0-10 NRS) after topical anesthesia placement. Individuals with persistent ocular pain also had worse dry eye symptoms (DEQ-5, $14.6 \pm$ 3.7 vs $12.7 \pm 3.3, \mathrm{p}=0.001)$ and photophobia intensity ( 5.6 \pm 3.1 vs $3.2 \pm 3.2, \mathrm{p}<0.0005,0-10 \mathrm{NRS}$ scale) compared to individuals without pain after topical anesthesia. ${ }^{77}$ These data highlight multiple clinical features suggestive of central abnormalities in individuals with dry eye symptoms. However, brain imaging studies would provide stronger evidence of central nerve abnormalities. While lacking for aqueous tear deficiency, a case report of functional magnetic resonance imaging (fMRI) in an individual with contact lens overuse (one contributor to dry eye) ${ }^{78}$ and photophobia reported activation at the level of the trigeminal ganglion, trigeminal nucleus caudalis, and thalamus when presented with 6-second blocks of light. ${ }^{65}$ The strength of this report is that it links corneal epithelial cell disruption to photophobia to activation of central trigeminal pathways. However, more imaging studies in a variety of dry eye sub-types are needed to supplement these findings. Quantitative sensory testing has also been applied to the study of dry eye, with higher dry eye symptoms associated with enhanced temporal summation and the presence of after-sensations, both of which suggest central contributions to symptoms. ${ }^{66}$

Similar to dry eye, central abnormalities have been found in individuals with migraine pain. ${ }^{79}$ In a Chinese study of 16 individuals with chronic migraine, 18 with episodic migraine, and 18 controls, individuals with chronic migraine demonstrated increased resting-state functional connectivity between bilateral amygdala and several brain regions compared to those with episodic migraine on fMRI. Compared to controls, those with chronic migraine had decreased functional connectivity between the right amygdala and several brain regions, whereas those with episodic migraine had increased functional connectivity in the left amygdala. ${ }^{80}$ In a Korean study, 19 individuals with chronic migraine had increased resting-state functional connectivity between pain processing areas and the dorsal raphe nucleus compared to 45 individuals with episodic migraine on fMRI. ${ }^{81}$ Together, these studies demonstrate central abnormities in animal models and humans with migraine, with greater abnormalities noted in individuals with chronic vs episodic migraine.

Inflammation is an important contributor to peripheral and central nerve abnormalities in dry eye and migraine.

Inflammatory mediators likely contribute to the development of peripheral and central sensitization in individuals with dry eye and migraine. For example, CGRP, a neuropeptide involved in neurogenic inflammation, as well as cardiovascular, gastrointestinal and endocrine processes, ${ }^{43}$ has been associated with changes in nerve function in dry eye and migraine. In a rat model of corneal abrasion using heptanol, CGRP increased in peripheral corneal nerves at one week (measurement at 24 hours was limited by the abrasion) and in the trigeminal ganglion at 24 hours compared to controls. Concomitantly, rats displayed corneal hyperalgesia (increased eye wipes after corneal application of menthol) at 24 hours compared to controls. Both CGRP levels and hyperalgesia decreased to baseline at 1 week. These results suggest an association between CGRP and peripheral nerve function. ${ }^{82}$

Inflammatory mediators have also been found to increase in the central nervous system in dry eye. In a mouse model of lacrimal gland excision, increased mRNA levels of pro-inflammatory markers were noted in the trigeminal ganglion and brainstem compared to sham controls 21 days post-surgery. Similar to the rat model, these mice also exhibited corneal hypersensitivity after injury. Additionally, increased spontaneous electrical activity in their ciliary nerve was noted compared to controls. Centrally, increased synaptic plasticity in the trigeminal brainstem complex (measured using immunofluorescence of presynaptic zone components) was observed at 21 days. ${ }^{70}$ This study demonstrates an association between aqueous tear deficiency, inflammation in central trigeminal pathways, and peripheral and central nerve abnormalities.

Human studies also support the link between inflammation and corneal nerve abnormalities. A Turkish study of 37 individuals with dry eye symptoms and signs (TBUT $<7$ seconds, corneal staining, Schirmer $<10 \mathrm{~mm}$ ) measured corneal sensitivity (via Cochet-Bonnet) before and after topical cyclosporine $0.05 \%$ (an antiinflammatory agent). Corneal sensitivity increased post vs pre cyclosporine therapy $(58.8 \pm 2.1$ vs $52.1 \pm$ $5.5 \mathrm{~mm}, \mathrm{p}<0.001) .{ }^{83}$ These data suggest that inflammation impacts corneal nerve sensitivity in dry eye.

Inflammation, specifically CGRP, has also been linked to nerve abnormalities in migraine. ${ }^{49}$ For example, a rat model 
of migraine (recurrent administration of nitroglycerin) found that CGRP-immunoreactive fibers significantly increased in the trigeminal nucleus caudalis compared to controls. This was clinically accompanied by thermal hyperalgesia (withdrawal latency after infrared radiation on hind paw). Furthermore, hyperalgesia was ameliorated by knocking down CGRP with short hairpin RNA. ${ }^{84}$ In a rat model of migraine (glass micropipette inserted into the visual cortex), a propagating wave of depolarization was induced with a resultant increase in the firing rate of spinal trigeminal nucleus neurons. ${ }^{85}$ The increased firing rate was blocked when rats were pretreated with a CGRP-blocking antibody. ${ }^{86}$ These data demonstrate that CGRP impacts nerve sensitivity in migraine.

CGRP has also been linked to migraine in humans. In a placebo-controlled, cross-over study of 13 individuals with migraine, intravenous CGRP induced migraine-like attacks in 10 individuals compared to 0 after placebo (isotonic saline), $\mathrm{p}=0.002$. Median peak headache intensity score (NRS scale 0 to 10 ) was 5 (5-9) after CGRP compared to $2(0-4)$ after placebo $(p=0.004) .{ }^{87}$ The effectiveness of anti-CGRP antibodies in treating migraine provides further support for the role of CGRP in migraine pathophysiology. ${ }^{88}$ Together, the above studies support the interaction between CGRP and nerve function in migraine.

\section{CGRP is Also Related to Light Sensitivity, Independent of Dry Eye and Migraine}

CGRP can induce light sensitivity. In wild-type mice, peripheral (intraperitoneal) and central (intracerebroventricular) injection of CGRP induced light-aversive behavior (time spent in illuminated portion of a light/dark box). Furthermore, an anti-CGRP monoclonal antibody attenuated light aversion after the peripheral injection of CGRP. $^{89}$ In transgenic mice that overexpressed the CGRP receptor, central, but not peripheral, CGRP administration induced light aversion. In another mouse model, peripheral injection of CGRP produced spontaneous pain (measured by a squint assay) both in complete darkness and in bright light. ${ }^{90}$ Together, these studies support the role of CGRP in pain and photophobia via multiple mechanisms.

\section{Light Can Trigger Corneal Inflammation and Nerve Abnormalities}

In a mouse model, blue light, but not yellow light, increased corneal sensitivity (via von Frey hair test) 3 hours post vs pre exposure. Exposure to blue light also led to observable changes on in-vivo confocal microscopy including activation of the superficial corneal epithelium (defined as the appearance of hyperreflective nuclei), increased numbers of dendritic (inflammatory) cells in the sub-basal plexus, and increased numbers of keratocytes in the stroma. ${ }^{91,92}$ Additionally, blue-light increased inflammation in both the trigeminal ganglia and spinal trigeminal nucleus, as measured by mRNA expression of cFOS and ATF3. ${ }^{46}$ These data suggest that the pathophysiology of dry eye and migraine is complex with multiple potential entry points (light, aqueous tear deficiency, corneal epithelial damage, cortical disruptions) that lead to inflammation and nerve abnormalities in multiple compartments (peripheral and central).

\section{Practical Implications for Diagnosing Dry Eye and Migraine}

The overlap between dry eye and migraine has potential implications in the evaluation and treatment of individuals with these two diseases as illustrated in Figure 2. First, eye care providers should ask individuals with dry eye about comorbid headache and primary care doctors and neurologists should ask individuals with migraine about symptoms of dry eye. If present, appropriate referrals can be made.

Second, given shared pathophysiology involving nerve dysfunction, eye care providers should think about nerve status when evaluating an individual with dry eye symptoms. This includes assessing for ocular pain via standardized questionnaires (eg NRS, Neuropathic Pain Symptom Inventory-Eye [NPSI-Eye]) and evaluating nerve structure and function clinically. The presence of cutaneous allodynia can be assessed by evaluating for pain to touch around the eyes. In addition, corneal sensitivity can be qualitatively checked with a cotton tip or dental floss (generally rated as $0=$ absent, $1=$ reduced, $2=$ normal, 3, increased). The proparacaine test can help differentiate between nociceptive pain ("pain that arises from actual or threatened damage to non-neural tissue and is due to activation of nociceptors") ${ }^{53}$ or peripheral neuropathic pain vs centrally mediated or non-ocular pain. ${ }^{77}$

Corneal nerves can be imaged with IVCM and certain nerve findings have been reported to suggest the presence of peripheral neuropathic pain. Specifically, one retrospective study found that in individuals with clinical suspected neuropathic pain, nerves in the subbasal layer abruptly terminated with hyperreflective enlargements. ${ }^{93}$ This finding was termed microneuroma based on similar findings in 


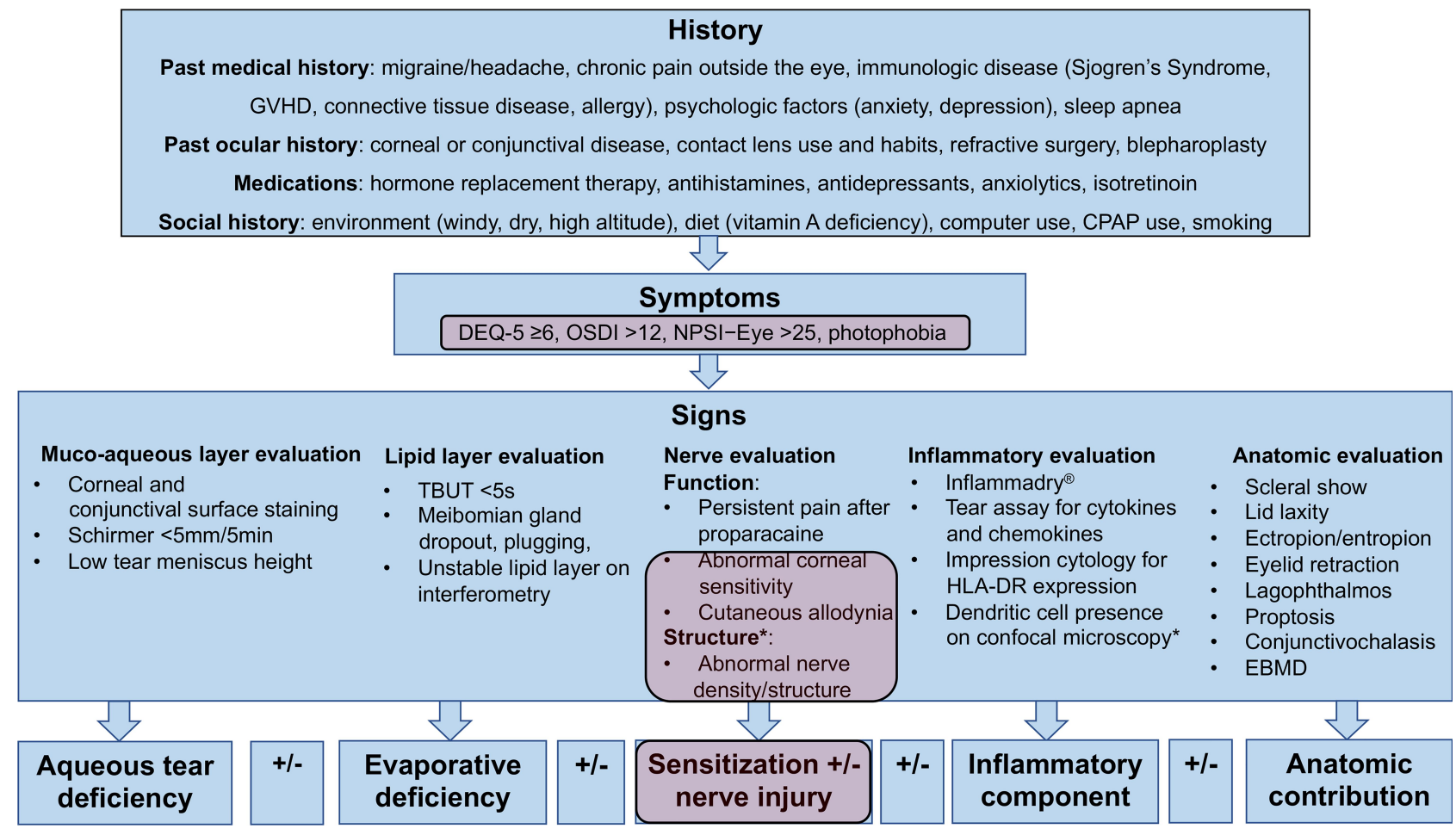

Figure 2 Clinical assessment of patients with dry eye. Purple boxes indicate phenotypes that overlap with migraine. *Nerve structure findings using in-vivo confocal microscopy.

Abbreviations: GVHD, graft versus host disease; DEQ-5, Dry Eye Questionnaire-5; OSDI, Ocular Surface Disease Index; NRS, numerical rating scale; NPSI-Eye, Neuropathic Pain Symptom Inventory-Eye; TBUT, tear break up time; EBMD, epithelial basement membrane dystrophy.

animal models. ${ }^{94}$ Microneuromas were observed in all subjects with ocular pain $(n=30)$, but were not present in any subjects without pain $(n=30) .{ }^{93}$ Other studies, however, failed to replicate these findings in other dry eye populations. ${ }^{95}$

Understanding nerve status in an individual patient may help explain their clinical presentation as different sensitivity profiles have been described in different dry eye populations (eg hyposensitivity in aqueous tear deficiency, hypersensitivity in individual with presumed neuropathic ocular pain ${ }^{96}$ and/or migraine). ${ }^{75}$ This heterogeneity may explain the disconnect often seen between dry eye symptoms and signs, as nerve function drives sensation, and thus symptomatic interpretation, of dry eye signs (decreased tear volume, rapid tear evaporation). Understanding nerve status can also help tailor an individualized treatment plan.

\section{An Updated Paradigm for the Treatment of Dry Eye Based on Data in Migraine}

The current paradigm for managing dry eye is to target tear dysfunction. This new paradigm suggests that when this approach does not sufficiently relieve dry eye symptoms, therapies targeting nerve dysfunction should be considered. Given similarities between dry eye and migraine, therapies that are of benefit in migraine may be beneficial in dry eye.

\section{Anti-Inflammatory Therapy}

Anti-inflammatory medications are a first-line treatment in dry eye and migraine ${ }^{69,97}$ Specifically, in dry eye, shortterm topical corticosteroids, and long-term cyclosporine and lifitegrast are first-line agents. ${ }^{67}$ Decreasing ocular surface inflammation may improve tear composition and dry eye symptoms. ${ }^{98}$ However, similar to migraine, ${ }^{99}$ not all patients with dry eye respond to anti-inflammatory therapy. ${ }^{100}$ Interestingly, baseline nerve status may predict who responds to anti-inflammatory therapy. In an American study, 60 individuals with dry eye (OSDI $>22$, corneal staining, meibomian gland dysfunction) were grouped by subbasal corneal nerve length $(<16.84(\mathrm{n}=9)$ vs $\geq 16.84 \mathrm{~mm} / \mathrm{mm}^{2}, \mathrm{n}=11$ ). Symptoms and signs in individuals with higher baseline SNFL improved 4 weeks after starting loteprednol (Symptom Assessment in Dry Eye, SANDE: $60.1 \pm 17.4$ vs $50.0 \pm 22.7, \mathrm{p}=0.04$ and corneal 
staining: $6.7 \pm 3.2$ vs $4.6 \pm 2.9, \mathrm{p}=0.01$ ) while those with low baseline nerve length showed no improvement. ${ }^{100}$ In patients who fail anti-inflammatory therapies, other therapies need to be considered.

\section{Oral Nerve Modulators}

In individuals with features suggestive of centrally mediated pain (peri-ocular allodynia to light touch, photophobia, persistent pain after anesthesia), systemic nerve modulators should be considered. Oral nerve modulators have been effective for migraine prevention including, serotonin and norepinephrine reuptake inhibitors and tricyclic antidepressants (TCAs), ${ }^{25}$ and for aborting acute migraine attacks, such as triptans. ${ }^{101}$ Given similar pathophysiology to migraine, patients with dry eye may also benefit from oral nerve modulators. Indeed, gabapentin and pregabalin, both alpha 2 delta $(\alpha 2 \gamma)$ ligands, have been examined in dry eye. These agents are thought to exert their effect by reducing voltage-gated calcium channel currents in the central nervous system leading to decreased excitatory neurotransmission. ${ }^{102}$ A case series evaluated the efficacy of $\alpha 2 \gamma$ ligands in 8 individuals with ocular pain unresponsive to topical therapies. Gabapentin was escalated to a dose of $600-900 \mathrm{mg}$ three times daily and pregabalin to $150 \mathrm{mg}$ twice in the study. Two individuals reported complete pain relief after adding a $\alpha 2 \gamma$ ligand to their multi-modal regimen while 3 individuals reported significant relief. ${ }^{102}$ Interestingly, the 2 individuals with complete pain relief were also on concomitant duloxetine. This study demonstrates that $\alpha 2 \gamma$ ligands may alleviate ocular pain in dry eye as part of a multi-modal regimen. However, additional studies are needed.

As with migraine, groups have studied the impact of TCAs in nerve-related ocular pain. TCAs inhibit central and peripheral serotonin and norepinephrine reuptake as well as cholinergic, histaminergic, and sodium channels. ${ }^{98}$ In a retrospective cohort study of 30 patients who failed other therapies and had persistent pain after anesthesia, nortriptyline (at least 4 weeks of use, started at $10 \mathrm{mg}$ and increased up to $100 \mathrm{mg}$ based on response and tolerability) improved ocular pain in the last 24-hours (measured via NRS) from $5.7 \pm 2.1$ to $3.6 \pm 2.1$ after $10.5 \pm 9.1$ months ( $\mathrm{p}<0.0001)$ of use. In addition, quality of life score (via an OPAS sub-score) improved from $6.0 \pm 2.5$ to $4.3 \pm$ $2.4(\mathrm{p}=0.019) .{ }^{103}$ Taken together, the above studies suggest that in individuals with dry eye symptoms and clinical features suggestive of central nerve abnormalities, oral nerve modulators may improve ocular pain symptoms.
However, in patients with either dry eye or migraine who show no or partial response to oral therapies, adjuvant approaches may be considered.

\section{Adjuvant Approaches}

Adjuvant therapies are often employed in migraine and may also be beneficial in the treatment of dry eye. For example, botulinum toxin is an approved medication in migraine ${ }^{104}$ and has been explored in dry eye. Botulinum toxin is thought to target pain responses by reducing facial muscle contraction and thus decreasing trigeminal afferent signaling as well as by reducing synaptic release of CGRP. ${ }^{42}$ In migraine, a Cochrane meta-analysis of 26 double-blind randomized controlled trials found that botulinum toxin treatment reduced the frequency of migraine (mean difference $=-2.39$ migraine days $/$ month; $95 \% \mathrm{CI},-4.02$ to -0.76 ) and migraine severity (measured by NRS $0-10$; mean difference $=-3.30 ; 95 \% \mathrm{CI},-4.16$ to -2.45$)$ compared to placebo in those with episodic or chronic migraine. ${ }^{105} \mathrm{In}$ dry eye, a retrospective study of 117 South Florida veterans with chronic migraine ( $\geq 15$ headaches or headache days/ month) found that botulinum toxin A (mean units injected: $114.4 \pm 24.5$ ) improved migraine pain (mean change $=$ $-3.43 ; 95 \% \mathrm{CI},-3.95$ to $-2.92 ; \mathrm{p}<0.001)$, photophobia (mean difference $=-2.64 ; 95 \% \mathrm{CI},-3.18$ to -2.11 ; $\mathrm{p}<0.001$ ), and dry eye symptoms (mean difference= $-0.716 ; 95 \% \mathrm{CI},-1.18$ to $-0.249 ; \mathrm{p}=0.003$ ) (all measured via NRS $0-10)$ compared to pre-injection scores. ${ }^{106}$ This effect was found to be independent of tear volume, ${ }^{42}$ suggesting that mechanisms beyond tear dysfunction drive eye symptoms. In 4 individuals with dry eye symptoms without migraine, a modified botulinum toxin A protocol (35 units in 7 sites) improved photophobia and dry eye symptoms 1 month post vs pre injection. ${ }^{107}$ Together, these data suggest that botulinum toxin A may improve photophobia and dry eye symptoms in individuals with and without migraine.

Another adjuvant treatment with success in migraine is device neuromodulation, and this entity has also been studied in dry eye. Specifically, transcutaneous electrical nerve stimulation (TENS) uses pulsed low voltage electrical currents across the intact surface of the skin to stimulate peripheral nerves. ${ }^{108}$ TENS has been postulated to improve pain by stimulating deep sensory afferents that secondarily inhibit nociceptive input via gate control theory. ${ }^{108}$ As applied to ocular pain, TENS may stimulate deep $A \beta$ fibers in the V1 and V2 distribution and block nociceptive input from unmyelinated $\mathrm{C}$ fibers. In terms of migraine, one meta-analysis of four studies using different 
TENS devices, Cefaly (company, location), LH202H Han Electrostimulator (company, location), GammaCore ${ }^{\circledR}$ (company, location), HANS-200A machine (company, location), with varying protocols (five times weekly, daily, three times daily) found that TENS significantly reduced monthly headache days (standard mean difference $=-0.48 ; 95 \% \mathrm{CI},-0.73$ to $-0.23 ; \mathrm{p}<0.001)$ and analgesic intake (standard mean difference $=-0.78 ; 95 \%$ CI, -1.14 to $-0.42 ; \mathrm{p}<0.001)$ compared to sham TENS (TENS device was applied with far less electrical stimulation or none at all). ${ }^{108}$ Similar to migraine, TENS has also shown promise in dry eye. In a retrospective study of 10 individuals with ocular pain, some of which had dry eye signs, an RS4i (RS medical, Vancouver) was used at varying intervals (range 3-21 times weekly) for a median of 6.5 months (range: 3-14 months). Overall, pain scores (one-week recall measured via NRS 0-10) decreased by $27.4 \%(\mathrm{p}=0.02)$ post- vs pre-treatment. ${ }^{109}$ Together, these data suggest that TENS may be incorporated as an adjuvant treatment in individuals with dry eye and migraine.

Another modality less frequently used in migraine is the blockage of peripheral nerve afferents with local anesthetic. ${ }^{102}$ In migraine, a meta-analysis of 33 articles showed that blockade of the greater occipital nerve was associated with a significant decrease in the number of headache days (pooled mean difference in headache days $=-3.6 ; 95 \% \mathrm{CI},-1.39$ to -5.81$)$ and headache severity (pooled mean difference in pain scores $=-2.2 ; 95 \% \mathrm{CI}$, -1.56 to -2.84$).{ }^{110}$ This approach may also benefit patients with ocular pain when applied to trigeminal nerve afferents. A retrospective series of eleven individuals who failed conservative therapy for dry eye and ocular pain reported outcomes after periocular nerve block with $4 \mathrm{~mL}$ of $0.5 \%$ bupivacaine mixed with $1 \mathrm{~mL}$ of $80 \mathrm{mg} / \mathrm{mL}$ methylprednisolone acetate targeting the supraorbital, supratrochlear, infratrochlear, and infraorbital nerves. Seven of 11 individuals experienced pain relief after nerve block lasting hours to months and five individuals underwent repeat nerve blocks. ${ }^{102}$ Of note, four of the seven individuals who responded to nerve blocks had ocular surgery as the pain trigger, whereas this was the case for one of the four non-responders. The above studies suggest that nerve blocks may benefit some patients with refractory ocular pain. However, these data are limited by their observational nature and limited number of subjects.

In addition to trigeminal afferent blockade, other nerve block sites have shown promise for treatment of migraine and dry eye, such as sphenopalatine ganglion (SPG) blocks. ${ }^{111,112}$ In fact, some ocular pain is thought to be mediated by parasympathetic fibers, whose presence has been documented on the cornea. ${ }^{113}$ Although biologic plausibility exists, studies are needed to evaluate the effects of SPG blocks in individuals with dry eye symptoms and ocular pain. Overall, the data presented in this section support the use of nerve blocks in appropriate individuals, especially those with surgically induced chronic ocular pain.

\section{Conclusions}

To conclude, this review discusses potential links between dry eye and migraine, prompted by an association between the two diseases in the literature. This information can be used to better understand pathophysiological mechanisms and develop targeted treatments by applying therapies successful in reducing migraine pain to dry eye. Neuronal injury leading to peripheral and central sensitization through trigeminal pathways are important mechanisms in some individuals with dry eye symptoms. Clinically, these individuals may manifest as hyperalgesia (evoked pain with wind), photophobia, and expansions of the receptive field (pain to light touch of the skin around the eye). These data highlight the need to test for nerve function in individuals with dry eye and consider the use of therapies that target nerve abnormalities in appropriate individuals.

\section{Disclosure}

Dr Anat Galor reports being supported by the Department of Veterans Affairs, Veterans Health Administration, Office of Research and Development, Clinical Sciences R\&D (CSRD) I01 CX002015 (Dr. Galor) and Biomedical Laboratory R\&D (BLRD) Service I01 BX004893 (Dr. Galor), Department of Defense Gulf War Illness Research Program (GWIRP) W81XWH-20-1-0579 (Dr. Galor) and Vision Research Program (VRP) W81XWH-20-1-0820 (Dr. Galor), National Eye Institute R01EY026174 (Dr. Galor) and R61EY032468 (Dr. Galor), NIH Center Core Grant P30EY014801 (institutional) and Research to Prevent Blindness Unrestricted Grant (institutional). The authors report no other potential conflicts of interest for this work.

\section{References}

1. McDonald M, Patel DA, Keith MS, Snedecor SJ. Economic and humanistic burden of dry eye disease in Europe, North America, and Asia: a systematic literature review. Ocul Surf. 2016;14(2):144-167. doi:10.1016/j.jtos.2015.11.002 
2. Younger DS. Epidemiology of migraine. Neurol Clin. 2016;34 (4):849-861. doi:10.1016/j.ncl.2016.06.011

3. Bonafede M, Sapra S, Shah N, Tepper S, Cappell K, Desai P. Direct and indirect healthcare resource utilization and costs among migraine patients in the United States. Headache. 2018;58(5):700-714. doi:10.1111/head.13275

4. Craig JP, Nichols KK, Akpek EK, et al. TFOS DEWS II definition and classification report. Ocul Surf. 2017;15(3):276-283. doi:10.1016/j.jtos.2017.05.008

5. Kalangara JP, Galor A, Levitt RC, et al. Characteristics of ocular pain complaints in patients with idiopathic dry eye symptoms. Eye Contact Lens. 2017;43(3):192-198. doi:10.1097/ICL.0000 00000000249

6. Stapleton F, Alves M, Bunya VY, et al. TFOS DEWS II epidemiology report. Ocul Surf. 2017;15(3):334-365.

7. Chalmers RL, Begley CG, Caffery B. Validation of the 5-Item Dry Eye Questionnaire (DEQ-5): discrimination across self-assessed severity and aqueous tear deficient dry eye diagnoses. Cont Lens Anterior Eye. 2010;33(2):55-60. doi:10. 1016/j.clae.2009.12.010

8. Schiffman RM, Christianson MD, Jacobsen G, Hirsch JD, Reis BL. Reliability and validity of the ocular surface disease index. Arch Ophthalmol. 2000;118(5):615-621. doi:10.1001/ archopht.118.5.615

9. Miller KL, Walt JG, Mink DR, et al. Minimal clinically important difference for the ocular surface disease index. Arch Ophthalmol. 2010;128(1):94-101. doi:10.1001/archophthalmol. 2009.356

10. Qazi Y, Hurwitz S, Khan S, Jurkunas UV, Dana R, Hamrah P. Validity and reliability of a Novel Ocular Pain Assessment Survey (OPAS) in quantifying and monitoring corneal and ocular surface pain. Ophthalmology. 2016;123(7):1458-1468. doi:10.1016/j. ophtha.2016.03.006

11. Farhangi M, Feuer W, Galor A, et al. Modification of the Neuropathic Pain Symptom Inventory for use in eye pain (NPSI-Eye). Pain. 2019;160(7):1541-1550. doi:10.1097/j.pain.00 00000000001552

12. Willcox MDP, Argueso P, Georgiev GA, et al. TFOS DEWS II tear film report. Ocul Surf. 2017;15(3):366-403.

13. Nelson JD, Craig JP, Akpek EK, et al. TFOS DEWS II Introduction. Ocul Surf. 2017;15(3):269-275. doi:10.1016/j.jtos. 2017.05.005

14. Chhadva P, Goldhardt R, Galor A. Meibomian gland disease: the role of gland dysfunction in dry eye disease. Ophthalmology. 2017;124(11):S20-S26. doi:10.1016/j.ophtha.2017.05.031

15. Wolffsohn JS, Arita R, Chalmers R, et al. TFOS DEWS II diagnostic methodology report. Ocul Surf. 2017;15(3):539-574.

16. Lanza NL, Valenzuela F, Perez VL, Galor A. The matrix metalloproteinase 9 point-of-care test in dry eye. Ocul Surf. 2016;14 (2):189-195. doi:10.1016/j.jtos.2015.10.004

17. Bartlett JD, Keith MS, Sudharshan L, Snedecor SJ. Associations between signs and symptoms of dry eye disease: a systematic review. Clin Ophthalmol (Auckland, NZ). 2015;9:1719-1730. doi:10.2147/OPTH.S89700

18. Ong ES, Felix ER, Levitt RC, Feuer WJ, Sarantopoulos CD, Galor A. Epidemiology of discordance between symptoms and signs of dry eye. Br J Ophthalmol. 2018;102(5):674-679. doi:10. 1136/bjophthalmol-2017-310633

19. Pouyeh B, Viteri E, Feuer W, et al. Impact of ocular surface symptoms on quality of life in a United States veterans affairs population. Am J Ophthalmol. 2012;153(6):1061-1066 e1063. doi:10.1016/j.ajo.2011.11.030

20. Schiffman RM, Walt JG, Jacobsen G, Doyle JJ, Lebovics G, Sumner W. Utility assessment among patients with dry eye disease. Ophthalmology. 2003;110(7):1412-1419. doi:10.1016/ S0161-6420(03)00462-7
21. Fernandez CA, Galor A, Arheart KL, et al. Dry eye syndrome, posttraumatic stress disorder, and depression in an older male veteran population. Invest Ophthalmol Vis Sci. 2013;54 (5):3666-3672. doi:10.1167/iovs.13-11635

22. Labbe A, Wang YX, Jie Y, Baudouin C, Jonas JB, Xu L. Dry eye disease, dry eye symptoms and depression: the Beijing Eye Study. Br J Ophthalmol. 2013;97(11):1399-1403. doi:10.1136/ bjophthalmol-2013-303838

23. Au NH, Mather R, To A, Malvankar-Mehta MS. Sleep outcomes associated with dry eye disease: a systematic review and meta-analysis. Can J Ophthalmol. 2019;54(2):180-189. doi:10. 1016/j.jcjo.2018.03.013

24. Headache Classification Committee of the International Headache Society (IHS). The international classification of headache disorders, 3rd edition. Cephalalgia. 2018;38(1):1-211. doi:10.1177/ 0333102417738202

25. Burch RC, Buse DC, Lipton RB. Migraine: epidemiology, Burden, and Comorbidity. Neurol Clin. 2019;37(4):631-649. doi:10.1016/j.ncl.2019.06.001

26. Leonardi M, Raggi A, Bussone G, D’Amico D. Health-related quality of life, disability and severity of disease in patients with migraine attending to a specialty headache center. Headache. 2010;50(10):1576-1586. doi:10.1111/j.1526-4610.2010.01770.x

27. Vo P, Fang J, Bilitou A, Laflamme AK, Gupta S. Patients' perspective on the burden of migraine in Europe: a crosssectional analysis of survey data in France, Germany, Italy, Spain, and the United Kingdom. J Headache Pain. 2018;19. doi:10.1186/s10194-018-0907-6

28. Ozudogru S, Neufeld A, Katz BJ, et al. Reduced visual quality of life associated with migraine is most closely correlated with symptoms of dry eye. Headache. 2019;59(10):1714-1721. doi:10. 1111/head.13662

29. Yang S, Kim W, Kim HS, Na KS. Association between migraine and dry eye disease: a nationwide population-based study. Curr Eye Res. 2017;42(6):837-841. doi:10.1080/02713683.2016.1262876

30. Ismail OM, Poole ZB, Bierly SL, et al. Association between dry eye disease and migraine headaches in a large population-based study. JAMA Ophthalmol. 2019;137(5):532-536. doi:10.1001/ jamaophthalmol.2019.0170

31. Wang TJ, Wang IJ, Hu CC, Lin HC. Comorbidities of dry eye disease: a nationwide population-based study. Acta Ophthalmol. 2012;90(7):663-668. doi:10.1111/j.1755-3768.2010.01993.x

32. Buse DC, Manack AN, Fanning KM, et al. Chronic migraine prevalence, disability, and sociodemographic factors: results from the American migraine prevalence and prevention study. Headache. 2012;52(10):1456-1470. doi:10.1111/j.1526-4610.20 12.02223.x

33. Farhangi M, Diel RJ, Buse DC, et al. Individuals with migraine have a different dry eye symptom profile than individuals without migraine. Br J Ophthalmol. 2020;104(2):260-264. doi:10.1136/ bjophthalmol-2018-313471

34. Koktekir BE, Celik G, Karalezli A, Kal A. Dry eyes and migraines: is there really a correlation? Cornea. 2012;31 (12):1414-1416. doi:10.1097/ICO.0b013e318247ec2a

35. Sarac O, Kosekahya P, Yildiz Tasci Y, et al. The prevalence of dry eye and sjogren syndrome in patients with migraine. Ocul Immunol Inflamm. 2017;25(3):370-375. doi:10.3109/09273948.2015.1132739

36. Kinard KI, Smith AG, Singleton JR, et al. Chronic migraine is associated with reduced corneal nerve fiber density and symptoms of dry eye. Headache. 2015;55(4):543-549. doi:10.1111/head.12547

37. Celikbilek A, Adam M. The relationship between dry eye and migraine. Acta Neurol Belg. 2015;115(3):329-333. doi:10.1007/ s13760-014-0359-y

38. Noseda R, Copenhagen D, Burstein R. Current understanding of photophobia, visual networks and headaches. Cephalalgia. 2018;39(13):1623-1634. doi:10.1177/0333102418784750 
39. Galor A, Levitt RC, Felix ER, Sarantopoulos CD. What can photophobia tell us about dry eye? Expert Rev Ophthalmol. 2016;11(5):321-324. doi:10.1080/17469899.2016.1222905

40. Galor A, Zlotcavitch L, Walter SD, et al. Dry eye symptom severity and persistence are associated with symptoms of neuropathic pain. Br J Ophthalmol. 2015;99(5):665-668. doi:10.1136/ bjophthalmol-2014-306057

41. Munjal S, Singh P, Reed ML, et al. Most bothersome symptom in persons with migraine: results from the Migraine in America Symptoms and Treatment (MAST) study. Headache. 2020;60 (2):416-429. doi:10.1111/head.13708

42. Diel RJ, Hwang J, Kroeger ZA, et al. Photophobia and sensations of dryness in migraine patients occur independent of baseline tear volume and improve following botulinum toxin A injections. $\mathrm{Br}$ $J$ Ophthalmol. 2019;103(8):1024-1029. doi:10.1136/bjophthalmol-2018-312649

43. Diel RJ, Mehra D, Kardon R, Buse DC, Moulton E, Galor A. Photophobia: shared pathophysiology underlying dry eye disease, migraine and traumatic brain injury leading to central neuroplasticity of the trigeminothalamic pathway. Br J Ophthalmol. 2020; bjophthalmol-2020-316417.

44. Brennan KC, Pietrobon D, A systems neuroscience approach to migraine. Neuron. 2018;97(5):1004-1021. doi:10.1016/j.neuron. 2018.01.029

45. Okamoto K, Thompson R, Tashiro A, Chang Z, Bereiter DA. Bright light produces Fos-positive neurons in caudal trigeminal brainstem. Neuroscience. 2009;160(4):858-864. doi:10.1016/j. neuroscience.2009.03.003

46. Marek V, Reboussin E, Dégardin-Chicaud J, et al. Implication of melanopsin and trigeminal neural pathways in blue light photosensitivity in vivo. Front Neurosci. 2019;13:497. doi:10.3389/ fnins.2019.00497

47. Burstein R, Noseda R, Fulton AB. Neurobiology of photophobia. $J$ Neuroophthalmol. 2019;39(1):94-102. doi:10.1097/WNO.000 0000000000766

48. Noseda R, Kainz V, Jakubowski M, et al. A neural mechanism for exacerbation of headache by light. Nat Neurosci. 2010;13 (2):239-245. doi:10.1038/nn.2475

49. Wattiez A-S, Sowers LP, Russo AF. Calcitonin gene-related peptide (CGRP): role in migraine pathophysiology and therapeutic targeting. Expert Opin Ther Targets. 2020;24(2):91-100. doi:10. 1080/14728222.2020.1724285

50. Sowers LP, Wang M, Rea BJ, et al. Stimulation of posterior thalamic nuclei induces photophobic behavior in mice Headache. 2020;60(9):1961-1981. doi:10.1111/head.13917

51. Bernstein CA, Nir -R-R, Noseda R, et al. The migraine eye: distinct rod-driven retinal pathways' response to dim light challenges the visual cortex hyperexcitability theory. Pain. 2019;160 (3):569-578. doi:10.1097/j.pain.0000000000001434

52. Xue T, Do MTH, Riccio A, et al. Melanopsin signalling in mammalian iris and retina. Nature. 2011;479(7371):67-73. doi: $10.1038 /$ nature 10567

53. IASP terminology; 2018. Available from: http://www.iasp-pain org/terminology. Accessed February 22, 2021.

54. Belmonte C. Pain, dryness, and itch sensations in eye surface disorders are defined by a balance between inflammation and sensory nerve injury. Cornea. 2019;38:S11-S24. doi:10.1097/ ICO.0000000000002116

55. Joubert F, Acosta M, Gallar J, et al. Effects of corneal injury on ciliary nerve fibre activity and corneal nociception in mice: a behavioural and electrophysiological study. Eur J Pain. 2019;23(3):589-602. doi:10.1002/ejp.1332

56. Kovács I, Luna C, Quirce S, et al. Abnormal activity of corneal cold thermoreceptors underlies the unpleasant sensations in dry eye disease. Pain. 2016;157(2):399-417. doi:10.1097/j.pain.0000 000000000455
57. Yang AY, Chow J, Liu J. Corneal innervation and sensation: the eye and beyond. Yale J Biol Med. 2018;91:13-21.

58. Parra A, Madrid R, Echevarria D, et al. Ocular surface wetness is regulated by TRPM8-dependent cold thermoreceptors of the cornea. Nat Med. 2010;16(12):1396-1399. doi:10.1038/nm.2264

59. Rahman M, Okamoto K, Thompson R, Katagiri A, Bereiter DA. Sensitization of trigeminal brainstem pathways in a model for tear deficient dry eye. PAIN. 2015;156(5):942-950. doi:10.1097/j. pain.0000000000000135

60. Mehra D, Cohen NK, Galor A. Ocular surface pain: a narrative review. Ophthalmol Ther. 2020;9(3):1-21. doi:10.1007/s40123020-00263-9

61. Galor A, Felix ER, Feuer W, Levitt RC, Sarantopoulos CD. Corneal nerve pathway function in individuals with dry eye symptoms. Ophthalmology. 2020. doi:10.1016/j.ophtha.2020.07. 061

62. Patel S, Hwang J, Mehra D, Galor A. Corneal nerve abnormalities in ocular and systemic diseases. Exp Eye Res. 2020;202:108284. doi:10.1016/j.exer.2020.108284

63. Kowtharapu BS, Winter K, Marfurt C, et al. Comparative quantitative assessment of the human corneal sub-basal nerve plexus by in vivo confocal microscopy and histological staining. Eye. 2017;31(3):481-490. doi:10.1038/eye.2016.220

64. Harte SE, Harris RE, Clauw DJ. The neurobiology of central sensitization. J Appl Biobehav Res. 2018;23(2):e12137.

65. Moulton EA, Becerra L, Borsook D. An fMRI case report of photophobia: activation of the trigeminal nociceptive pathway. Pain. 2009;145(3):358-363. doi:10.1016/j.pain.2009.07.018

66. Galor A, Levitt RC, McManus KT, et al. Assessment of somatosensory function in patients with idiopathic dry eye symptoms. JAMA Ophthalmol. 2016;134(11):1290-1298. doi:10.1001/ jamaophthalmol.2016.3642

67. Galor A, Moein H-R, Lee C, et al. Neuropathic pain and dry eye. Ocul Surf. 2018;16:31-44. doi:10.1016/j.jtos.2017.10.001

68. Burstein R, Noseda R, Borsook D. Migraine: multiple processes, complex pathophysiology. J Neurosci. 2015;35(17):6619-6629. doi:10.1523/JNEUROSCI.0373-15.2015

69. Charles A. The pathophysiology of migraine: implications for clinical management. Lancet Neurol. 2018;17(2):174-182. doi:10.1016/S1474-4422(17)30435-0

70. Fakih D, Zhao Z, Nicolle P, et al. Chronic dry eye induced corneal hypersensitivity, neuroinflammatory responses, and synaptic plasticity in the mouse trigeminal brainstem. $J$ Neuroinflammation. 2019;16(1):268. doi:10.1186/s12974-0191656-4

71. Giannaccare G, Pellegrini M, Sebastiani S, Moscardelli F, Versura P, Campos EC. In vivo confocal microscopy morphometric analysis of corneal subbasal nerve plexus in dry eye disease using newly developed fully automated system. Graefes Arch Clin Exp Ophthalmol. 2019;257(3):583-589. doi:10.1007/ s00417-018-04225-7

72. Rahman EZ, Lam PK, Chu C-K, Moore Q, Pflugfelder SC. Corneal sensitivity in tear dysfunction and its correlation with clinical parameters and blink rate. Am J Ophthalmol. 2015;160 (5):858-866.e855. doi:10.1016/j.ajo.2015.08.005

73. Shen F, Dong X, Zhou X, Yan L, Wan Q. Corneal subbasal nerve plexus changes in patients with episodic migraine: an in vivo confocal microscopy study. J Pain Res. 2019;12:1489-1495. doi:10.2147/JPR.S196705

74. Shetty R, Deshmukh R, Shroff R, Dedhiya C, Jayadev C. Subbasal nerve plexus changes in chronic migraine. Cornea. 2018;37(1):72-75. doi:10.1097/ICO.0000000000001403

75. Aykut V, Elbay A, Esen F, Kocaman G, Savran Elibol E, Oguz H. Patterns of altered corneal sensation in patients with chronic migraine. Eye Contact Lens. 2018;44:S400-S403. doi:10.1097/ ICL.0000000000000553 
76. Burstein R, Jakubowski M, Garcia-Nicas E, et al. Thalamic sensitization transforms localized pain into widespread allodynia. Ann Neurol. 2010;68(1):81-91. doi:10.1002/ana.21994

77. Crane AM, Feuer W, Felix ER, et al. Evidence of central sensitisation in those with dry eye symptoms and neuropathic-like ocular pain complaints: incomplete response to topical anaesthesia and generalised heightened sensitivity to evoked pain. $\mathrm{Br}$ $J$ Ophthalmol. 2017;101(9):1238-1243. doi:10.1136/bjophthalmol-2016-309658

78. Gomes JAP, Azar DT, Baudouin C, et al. TFOS DEWS II iatrogenic report. Ocul Surf. 2017;15(3):511-538.

79. Filippi M, Messina R. The chronic migraine brain: what have we learned from neuroimaging? Front Neurol. 2020;10:1356. doi:10.3389/fneur.2019.01356

80. Chen Z, Chen X, Liu M, Dong Z, Ma L, Yu S. Altered functional connectivity of amygdala underlying the neuromechanism of migraine pathogenesis. J Headache Pain. 2017;18(1):7. doi:10.11 86/s10194-017-0722-5

81. Lee MJ, Park B-Y, Cho S, Kim ST, Park H, Chung C-S. Increased connectivity of pain matrix in chronic migraine: a resting-state functional MRI study. J Headache Pain. 2019;20(1):29. doi:10.11 86/s10194-019-0986-Z

82. Hegarty DM, Hermes SM, Morgan MM, Aicher SA. Acute hyperalgesia and delayed dry eye after corneal abrasion injury. Pain Rep. 2018;3(4):e664-e664. doi:10.1097/PR9.000000000 0000664

83. Toker E, Asfuroglu E. Corneal and conjunctival sensitivity in patients with dry eye: the effect of topical cyclosporine therapy. Cornea. 2010;29(2):133-140. doi:10.1097/ICO.0b013e3181acf $68 \mathrm{~d}$

84. Zhang Y, Zhang Y, Tian K, et al. Calcitonin gene-related peptide facilitates sensitization of the vestibular nucleus in a rat model of chronic migraine. J Headache Pain. 2020;21(1):72. doi:10.1186/ s10194-020-01145-y

85. Zhang X, Levy D, Kainz V, Noseda R, Jakubowski M, Burstein R. Activation of central trigeminovascular neurons by cortical spreading depression. Ann Neurol. 2011;69(5):855-865. doi:10.1002/ana.22329

86. Melo-Carrillo A, Noseda R, Nir -R-R, et al. Selective inhibition of trigeminovascular neurons by fremanezumab: a humanized monoclonal anti-CGRP antibody. $J$ Neurosci. 2017;37 (30):7149-7163. doi:10.1523/JNEUROSCI.0576-17.2017

87. Christensen CE, Younis S, Deen M, Khan S, Ghanizada H, Ashina M. Migraine induction with calcitonin gene-related peptide in patients from erenumab trials. J Headache Pain. 2018;19 (1):105. doi:10.1186/s10194-018-0927-2

88. Urits I, Jones MR, Gress K, et al. CGRP antagonists for the treatment of chronic migraines: a comprehensive review. Curr Pain Headache Rep. 2019;23(5):29. doi:10.1007/s11916-019-07 68-y

89. Mason BN, Kaiser EA, Kuburas A, et al. Induction of migraine-like photophobic behavior in mice by both peripheral and central CGRP mechanisms. J Neurosci. 2017;37(1):204-216. doi:10.1523/JNEUROSCI.2967-16.2016

90. Rea BJ, Wattiez A-S, Waite JS, et al. Peripherally administered calcitonin gene-related peptide induces spontaneous pain in mice: implications for migraine. Pain. 2018;159(11):2306-2317. doi:10.1097/j.pain.0000000000001337

91. Aggarwal S, Kheirkhah A, Cavalcanti BM, Cruzat A, Jamali A, Hamrah P. Correlation of corneal immune cell changes with clinical severity in dry eye disease: an in vivo confocal microscopy study. Ocul Surf. 2020;19:183-189. doi:10.1016/j.jtos. 2020.05.012

92. Matsumoto Y, Ibrahim OMA. Application of in vivo confocal microscopy in dry eye disease. Invest Ophthalmol Vis Sci. 2018;59(14):DES41-DES47. doi:10.1167/iovs.17-23602
93. Moein H-R, Akhlaq A, Dieckmann G, et al. Visualization of microneuromas by using in vivo confocal microscopy: an objective biomarker for the diagnosis of neuropathic corneal pain? Ocul Surf. 2020;18(4):651-656. doi:10.1016/j.jtos.2020.07.004

94. Stepp MA, Pal-Ghosh S, Downie LE, et al. Corneal epithelial "neuromas": a case of mistaken identity? Cornea. 2020;39 (7):930-934. doi:10.1097/ICO.0000000000002294

95. Dermer H, Hwang J, Mittal R, Cohen A, Galor A. Corneal sub-basal nerve plexus microneuromas in individuals with and without dry eye. $\mathrm{Br} J$ Ophthalmol. 2021;bjophthalmol-2020317891. doi:10.1136/bjophthalmol-2020-317891

96. Spierer O, Felix ER, McClellan AL, et al. Corneal mechanical thresholds negatively associate with dry eye and ocular pain symptoms. Invest Ophthalmol Vis Sci. 2016;57(2):617-625. doi:10.1167/iovs.15-18133

97. Silbert JA, Bitton E, Bhagat K. Advances in diagnosis and management of dry eye disease. Adv Ophthalmol Optom. 2019;4:13-38. doi:10.1016/j.yaoo.2019.04.002

98. Dieckmann G, Goyal S, Hamrah P. Neuropathic corneal pain: approaches for management. Ophthalmology. 2017;124(11):S34s47. doi:10.1016/j.ophtha.2017.08.004

99. Ong JJY, De Felice M. Migraine treatment: current acute medications and their potential mechanisms of action. Neurotherapeutics. 2018;15(2):274-290. doi:10.1007/s13311-017-0592-1

100. Kheirkhah A, Dohlman TH, Amparo F, et al. Effects of corneal nerve density on the response to treatment in dry eye disease. Ophthalmology. 2015;122(4):662-668. doi:10.1016/j.ophtha.20 14.11 .006

101. Konstantinos S, Vikelis M, Rapoport A. Acute care and treatment of migraine. J Neuro Ophthalmol. 2020;40(4):472-484. doi:10.10 97/WNO.0000000000001053

102. Small LR, Galor A, Felix ER, Horn DB, Levitt RC, Sarantopoulos CD. Oral gabapentinoids and nerve blocks for the treatment of chronic ocular pain. Eye Contact Lens. 2020;46 (3):174-181. doi:10.1097/ICL.0000000000000630

103. Ozmen MC, Dieckmann G, Cox SM, et al. Efficacy and tolerability of nortriptyline in the management of neuropathic corneal pain. Ocul Surf. 2020;18(4):814-820. doi:10.1016/j.jtos.2020.08.006

104. Janis JE, Barker JC, Palettas M. Targeted peripheral nerve-directed onabotulinumtoxin A injection for effective long-term therapy for migraine headache. Plast Reconstr Surg Glob Open. 2017;5(3): e1270. doi:10.1097/GOX.0000000000001270

105. Herd CP, Tomlinson CL, Rick C, et al. Cochrane systematic review and meta-analysis of botulinum toxin for the prevention of migraine. BMJ Open. 2019;9(7):e027953. doi:10.1136/bmjopen-2018-027953

106. Diel RJ, Kroeger ZA, Levitt RC, et al. Botulinum toxin A for the treatment of photophobia and dry eye. Ophthalmology. 2018;125 (1):139-140. doi:10.1016/j.ophtha.2017.09.031

107. Venkateswaran N, Hwang J, Rong AJ, et al. Periorbital botulinum toxin A improves photophobia and sensations of dryness in patients without migraine: case series of four patients. Am J Ophthalmol Case Rep. 2020;19:100809. doi:10.1016/j.ajoc.2020.100809

108. Tao H, Wang T, Dong X, Guo Q, Xu H, Wan Q. Effectiveness of transcutaneous electrical nerve stimulation for the treatment of migraine: a meta-analysis of randomized controlled trials. J Headache Pain. 2018;19(1):42. doi:10.1186/s10194-018-0868-9

109. Zayan K, Aggarwal S, Felix E, Levitt R, Sarantopoulos K, Galor A. Transcutaneous electrical nerve stimulation for the long-term treatment of ocular pain. Neuromodulation. 2020;23 (6):871-877. doi:10.1111/ner.13146

110. Shauly O, Gould DJ, Sahai-Srivastava S, Patel KM. Greater occipital nerve block for the treatment of chronic migraine headaches: a systematic review and meta-analysis. Plast Reconstr Surg. 2019;144(4):943-952. doi:10.1097/PRS.0000000000006 059 
111. Cady R, Saper J, Dexter K, Manley HR. A double-blind, placebo-controlled study of repetitive transnasal sphenopalatine ganglion blockade with $\mathrm{tx} 360((\mathrm{R}))$ as acute treatment for chronic migraine. Headache. 2015;55(1):101-116. doi:10.1111/head.12458

112. Mehta D, Leary MC, Yacoub HA, et al. The effect of regional anesthetic sphenopalatine ganglion block on self-reported pain in patients with status migrainosus. Headache. 2019;59(1):69-76. doi:10.1111/head.13390
113. Chen W, Batawi HIM, Alava JR, et al. Bulbar conjunctival microvascular responses in dry eye. Ocul Surf. 2017;15 (2):193-201. doi:10.1016/j.jtos.2016.12.002

Eye and Brain

\section{Publish your work in this journal}

Eye and Brain is an international, peer-reviewed, open access journal focusing on clinical and experimental research in the field of neuroophthalmology. All aspects of patient care are addressed within the journal as well as basic research. Papers covering original research, basic science, clinical and epidemiological studies, reviews and

Submit your manuscript here: https://www.dovepress.com/eye-and-brain-journal evaluations, guidelines, expert opinion and commentary, case reports and extended reports are welcome. The manuscript management system is completely online and includes a very quick and fair peerreview system, which is all easy to use. Visit http://www.dovepress. com/testimonials.php to read real quotes from published authors. 